\title{
A bumblebee thioredoxin-like protein gene that is up-regulated by a temperature stimulus and lipopolysaccharide injection
}

\author{
Yong Soo CHOI ${ }^{1}$, Kwang Sik LEE ${ }^{1}$, Hyung Joo YOON ${ }^{2}$, Iksoo KIM², Hung Dae SOHN ${ }^{1}$ and Byung Rae JIN ${ }^{1 *}$ \\ ${ }^{1}$ College of Natural Resources and Life Science, Dong-A University, Busan 604-714, Korea \\ ${ }^{2}$ Department of Agricultural Biology, National Institute of Agricultural Science and Technology, Suwon 441-100, Korea
}

Key words. Antioxidant enzyme, Apidae, bumblebee, Bombus ignitus, gene structure, oxidative stress, reactive oxygen species (ROS), thioredoxin-like protein (txl)

\begin{abstract}
A thioredoxin-like protein (txl) gene was cloned from the bumblebee, Bombus ignitus. The B. ignitus txl (Bitxl) gene spans $1777 \mathrm{bp}$ and consists of three introns and four exons coding for 285 amino acid residues with a conserved active site (CGPC). The deduced amino acid sequence of the Bitxl cDNA was $65 \%$ similar to the Drosophila melanogaster txl. Northern blot analysis revealed the presence of Bitxl transcripts in all tissues examined. When $\mathrm{H}_{2} \mathrm{O}_{2}$ was injected into the body cavity of $B$. ignitus workers, Bitxl mRNA expression was up-regulated in the fat body tissue. In addition, the expression levels of Bitxl mRNA in the fat body greatly increased when B. ignitus workers were exposed to low $\left(4^{\circ} \mathrm{C}\right)$ or high $\left(37^{\circ} \mathrm{C}\right)$ temperatures, or injected with lipopolysaccharide (LPS), which suggests that the Bitxl possibly protects against oxidative stress caused by extreme temperatures and bacterial infection.
\end{abstract}

\section{INTRODUCTION}

Thioredoxins (Trx) are small thiol proteins with a molecular mass of about $12 \mathrm{kDa}$ that are evolutionarily conserved from prokaryotes to higher eukaryotes (Laurent et al., 1964; Holmgren, 1985, 1989). Trx contains two cysteine residues within the conserved active site sequence (CGPC) and many Trx-like proteins are members of the Trx superfamily (Nakamura, 2005). Trx functions as a hydrogen donor for many protein targets and a scavenger of reactive oxygen species (ROS) (Laurent et al., 1964; Holmgren, 1989; Arner \& Holmgren, 2000).

Several mammalian proteins of the Trx superfamily are known, which include Trx2 (Spyrou et al., 1997) and TRP32 (Lee et al., 1998). Trx2 is a mitochondrial protein with an active site, CGPC that acts as an electron donor for mitochondrial Trx-dependent peroxidase (Spyrou et al., 1997; Araki et al., 1999). TRP32 is a cytosolic protein with an active site, CGPC. The active site sequences of Trx 2 and TRP32 are identical to that of Trx. In addition, some other proteins contain a Trx domain, in which some residues within the active site are changed. Thioredoxinrelated transmembrane protein, TMX, possesses one TrXlike domain with a unique potentially active site sequence, CPAC, and bacterially expressed TMX shows Trx-like reducing activity in vitro (Matsuo et al., 2001). The Trx-like domain is also present in a nuclear protein termed nucleoredoxin with a modified active site sequence, CPPC (Kurooka et al., 1997).

A novel member of the expanding Trx superfamily, thioredoxin-like protein (txl) has been reported from man (Miranda-Vizuete et al., 1998). The human txl contains an extension of 184 residues at the C-terminus of the Trx domain (CGPC) and is not a substrate for thioredoxin reductase. Lee et al. (1998) identified the same protein (which they named TRP32) copurifying with a kinase that is proteolytically activated by caspases in apoptosis. The txl homologues identified from Drosophila melanogaster and Caenorhabditis elegans display much closer homology to the known Trxs than the human txl protein (Miranda-Vizuete \& Spyrou, 2000). Furthermore, critical residues for optimal Trx activity are present in both Drosophila and Caenorhabditis txl but absent in human txl, suggesting that txl might have evolved to carry out a function different from the general disulfide reductase typical of Trxs. Recently, however, metabolic enzymes of mycobacteria linked to antioxidant defense by a Trx-like protein were reported (Bryk et al., 2002). A Trx-like protein encoded by the $C$. elegans $d p y-11$ gene is required for morphogenesis (Ko \& Chow, 2002). These txl proteins seem to be involved in various redox regulations, but their biological functions are poorly understood.

With the aim of furthering the understanding of the role of insect $t x l$ gene in oxidative stress, txl up-regulation was analyzed under conditions that promote an increase in the levels of ROS. This paper describes the gene structure and characterization of the $t x l$ gene from the bumblebee, Bombus ignitus, which is an important pollinator of various greenhouse crops. To gain an insight into the physiological roles of insect $t x l$, the transcriptional induction of $B$. ignitus txl (Bitxl) in vivo by $\mathrm{H}_{2} \mathrm{O}_{2}$ or external low- and high-temperature stimuli was explored. The response of Bitxl to lipopolysaccharide (LPS), a major cell wall constituent of gram-negative bacterial organisms was also examined.

\footnotetext{
* Corresponding author; e-mail: brjin@dau.ac.kr
} 


\section{MATERIAL AND METHODS}

\section{Animals}

The bumblebee, Bombus ignitus, was reared under artificial conditions as described previously (Yoon et al., 2002, 2004).

\section{cDNA library screening, nucleotide sequencing and data analysis}

A cDNA library used in this study was constructed using whole bodies of $B$. ignitus workers. The clones harbouring cDNA inserts were randomly selected and sequenced to generate the expressed sequence tags (ESTs). The plasmid DNA was extracted by Wizard mini-preparation kit (Promega, Madison, WI). The nucleotide sequence was determined by using a BigDyeTerminator cycle sequencing kit in the automated DNA sequencer (model 310 Genetic Analyzer; Perkin-Elmer Applied Biosystems, Foster City, CA). The sequences were compared using the DNASIS and BLAST programs provided by the NCBI (http://www.ncbi.nlm.nih.gov/BLAST). GenBank, EMBL and SwissProt databases were searched for sequence homology using a BLAST algorithm program (Altschul et al., 1997). MacVector (ver. 6.5, Oxford Molecular Ltd) was used to align the amino acid sequences of txl.

\section{Genomic DNA isolation and PCR of the $t x l$ gene}

Genomic DNA was extracted from the fat body tissues of $B$. ignitus workers using a Wizard ${ }^{\mathrm{TM}}$ Genomic DNA Purification Kit, according to the manufacturer's instructions (Promega). The primers used for amplification of the genomic DNA encoding the txl were 5'-CGCTATAAAAATACAATGGGT GC-3' for the translational start sequence region and 5'ATAATCCTTTTAATGACTCTCCC-3' for the 3' non-coding region, based on the Bitxl cDNA cloned in this study. After a 35 -cycle amplification $\left(94^{\circ} \mathrm{C}\right.$ for $30 \mathrm{~s} ; 48^{\circ} \mathrm{C}$ for $40 \mathrm{~s} ; 72^{\circ} \mathrm{C}$ for 2 $\mathrm{min})$, PCR products were ethanol precipitated, centrifuged at $10,000 \times g$ for $15 \mathrm{~min}$, and rinsed with $70 \%$ ethanol. These DNAs were analyzed using $1.0 \%$ agarose gel electrophoresis. The PCR products for sequencing were cloned into pGem-T vector (Promega). The construct was transformed into Escherichia coli TOP10F' cells (Invitrogen, Carlsbad, CA). The nucleotide sequence was determined by using a BigDyeTerminator cycle sequencing kit and an automated DNA sequencer as described above.

\section{RNA isolation and Northern blot analysis}

Five B. ignitus workers were dissected on ice under a Stereomicroscope (Zeiss, Jena, Germany), samples of the fat body, midgut, muscle and head were collected, and washed twice with PBS (140 mM NaCl, $27 \mathrm{mM} \mathrm{KCl}, 8 \mathrm{mM} \mathrm{Na} \mathrm{HPO}_{4}, 1.5 \mathrm{mM}$ $\mathrm{KH}_{2} \mathrm{PO}_{4}, \mathrm{pH}$ 7.4). Total RNA was isolated from the fat body, midgut, muscle and head of $B$. ignitus workers using the Total RNA Extraction Kit (Promega). Total RNA (10 $\mu \mathrm{g} /$ lane) from B. ignitus workers was denatured by glyoxalation (McMaster \& Carmichael, 1977), transferred onto a nylon blotting membrane (Schleicher \& Schuell, Dassel, Germany) and hybridized at $42^{\circ} \mathrm{C}$ with a probe in a hybridization buffer containing $5 \times \mathrm{SSC}$, $5 \times$ Denhardt's solution, $0.5 \%$ SDS, and $100 \mu \mathrm{g} / \mathrm{ml}$ denatured salmon sperm DNA. The 855 bp Bitxl cDNA clone was labeled with $\left[\alpha{ }^{-32} \mathrm{P}\right] \mathrm{dCTP}$ (Amersham, Arlington Heights, IL), using the Prime-It II Random Primer Labeling Kit (Stratagene, La Jolla, $\mathrm{CA}$ ), and used as a probe for hybridization. After hybridization, the membrane filter was washed three times for $30 \mathrm{~min}$ each in $0.1 \%$ SDS and $0.2 \times \mathrm{SSC}(1 \times \mathrm{SSC}$ is $0.15 \mathrm{M} \mathrm{NaCl}$ and $0.015 \mathrm{M}$ sodium citrate) at $65^{\circ} \mathrm{C}$ and then exposed to autoradiography film.

\section{Txl expression in vivo after $\mathrm{H}_{2} \mathrm{O}_{2}$ treatment}

$B$. ignitus workers were injected with $10 \mathrm{mM} \mathrm{H}_{2} \mathrm{O}_{2}$. Three $B$. ignitus workers treated with $\mathrm{H}_{2} \mathrm{O}_{2}$ were dissected on ice under a microscope at $1 \mathrm{~h}$ intervals. The fat body tissues were harvested and washed twice with PBS. Total RNA was isolated from the fat body using the Total RNA Extraction Kit (Promega). Transcriptional induction of Bitxl was analyzed by Northern blot hybridization as described above.

\section{Txl expression in vivo after temperature treatment or lipo- polysaccharide (LPS) injection}

The B. ignitus workers were exposed to $4^{\circ} \mathrm{C}$ or $37^{\circ} \mathrm{C}$ for $6 \mathrm{~h}$, respectively, with control maintained at $27^{\circ} \mathrm{C}$. After incubation, fat body tissues from three $B$. ignitus workers were collected at $1 \mathrm{~h}$ intervals and washed twice with PBS. In addition, B. ignitus workers were injected with $10 \mathrm{mM}$ LPS (Sigma Chemical Co.). After treatment, fat body tissues from three $B$. ignitus workers treated with LPS were collected at $1 \mathrm{~h}$ intervals and washed twice with PBS. Total RNA was isolated from the fat body as described above. Induction of Bitxl transcripts in response to change in external temperature and LPS injection was analyzed by Northern blot as described above. Images of Northern blot were analyzed using a computerized image analysis system (Alpha Innotech Co., San Leandro, CA). Alpha Imager 1220

(A)

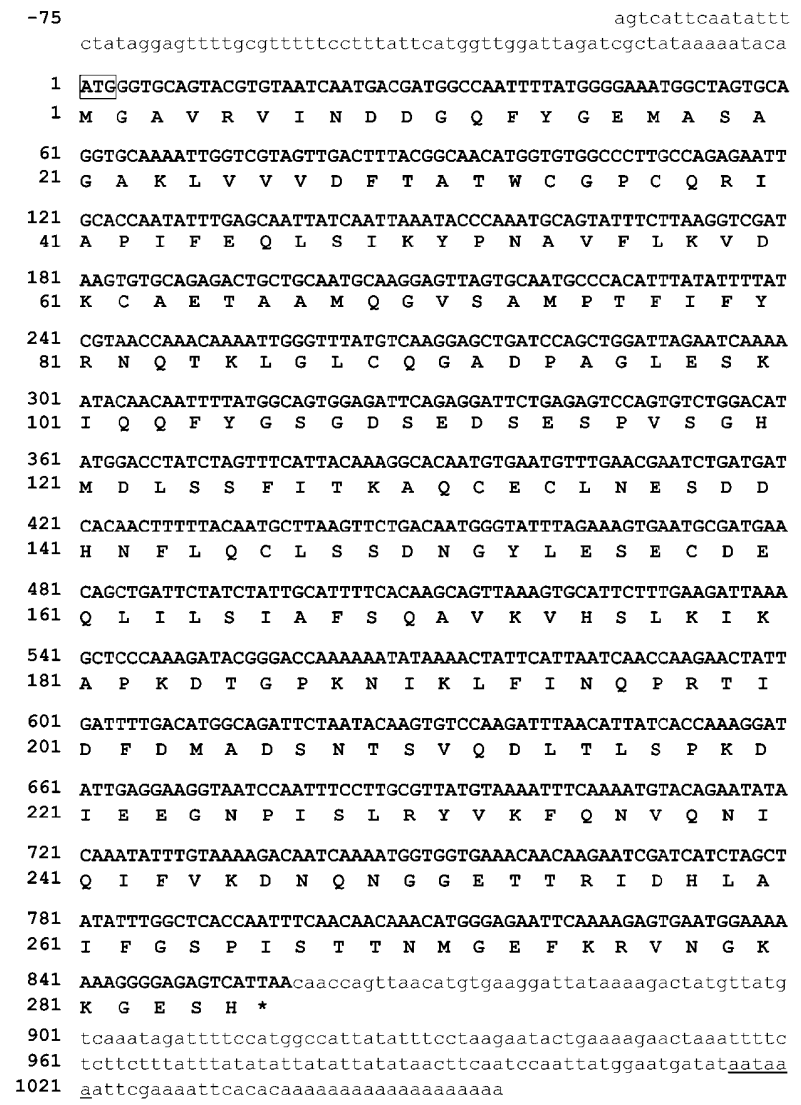

Fig. 1. cDNA sequence and structure of the Bitxl gene. (A) The nucleotide and deduced protein sequence of the Bitxl cDNA. The ATG start codon is boxed and the termination codon is indicated by an asterisk. In the cDNA sequence, the polyadenylation sequence is underlined. The GenBank accession number is DQ096568. (B) Organization of the Bitxl gene. Numbers indicate the position in the genomic sequences. The GenBank accession number is DQ096570. 
(B)

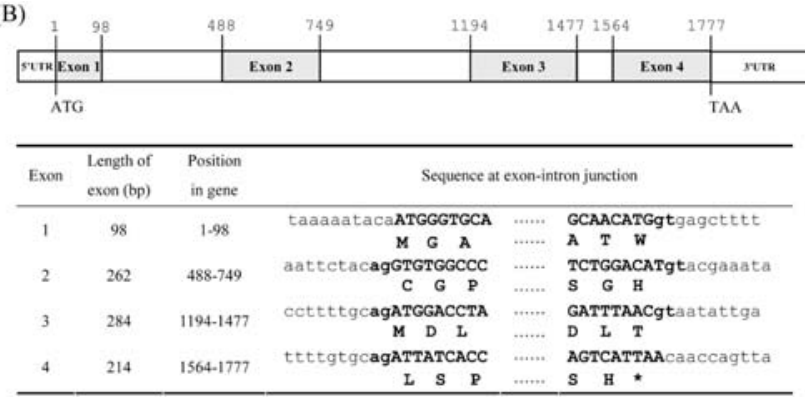

Fig. 1 continued.

(ver. 5.5) was used to aid the analyses. The integrated density value was used to determine the area of each band.

\section{RESULTS}

\section{Cloning, sequencing and analysis of Bitxl gene}

In search of B. ignitus ESTs, a cDNA was identified that had a high homology with previously reported $t x l$ genes. The cDNA clone, including the full-length open reading frame (ORF), was sequenced and characterized. The nucleotide and deduced amino acid sequences of the cDNA encoding $t x l$ are presented in Fig. 1A. The $B$. ignitus txl (Bitxl) cDNA is 1102 bp long and contains an ORF of 855 nucleotides capable of encoding a 285 amino acid polypeptide with a predicted molecular mass of $31298 \mathrm{Da}$ and $\mathrm{pI}$ of 4.83 . The ORF had both a start (ATG) and stop codon (TAA), indicating that the sequences contain the complete coding region. A putative polyadenylation signal, AATAAA, is located at nucleotide position 1016-1021.

To identify the genomic structure of the Bitxl gene, a primer set based on the sequences of the Bitxl cDNA was designed and a band was amplified from $B$. ignitus genomic DNA using this primer set. The PCR product was cloned and sequenced. Genomic PCR product sequences were $100 \%$ identical with Bitxl cDNA. The organization of the gene is illustrated in Fig. 1B. Comparison of the genomic sequence with the sequence of the cDNA revealed the presence of four exons and three introns in the Bitxl. The sequences at the exon-intron boundaries conformed the typical eukaryotic splice sites, including an invariant GT at the intron 5' boundary and an invariant AG at its 3' boundary. The genomic DNA size from translation start codon to stop codon was 1777 bp for Bitxl.

Comparison of the deduced amino acid sequence of the Bitxl with that of other txl sequences is shown in Fig. 2. The N-terminal part of the Bitxl protein contains the active site sequence, WCGPC, very similar to that found in most Trxs. Among the known txl sequences, Bitxl was closest to that of Drosophila melanogaster (65\% protein sequence identity) and relatively close to that of man (49\% identity) and Caenorhabditis elegans txl (45\% identity).

\section{Txl expression in B. ignitus tissues}

To characterize the expression of the Bitxl gene at the transcriptional level, Northern blot analysis was performed using total RNA obtained from fat body, midgut, muscle and head, respectively. Northern blot analysis showed that a hybridization signal was present in all these tissues, although the signal was weaker in the muscle than in the fat body and midgut (Fig. 3).

\section{$T \boldsymbol{x} l$ expression in vivo after $\mathrm{H}_{2} \mathrm{O}_{2}$ treatment}

When $\mathrm{H}_{2} \mathrm{O}_{2}$ was injected into body cavity of $B$. ignitus workers, the transcript level of Bitxl was assessed in total RNA isolated from the fat body (Fig. 4). As expected, the transcript level of Bitxl was significantly increased in the fat body after $1 \mathrm{~h}$, reached the highest level at $2 \mathrm{~h}$ and recovered after $7 \mathrm{~h}$, indicating that the Bitxl gene is upregulated in the presence of an $\mathrm{H}_{2} \mathrm{O}_{2}$ challenge.

\section{$\boldsymbol{T} \boldsymbol{x} \boldsymbol{l}$ expression in vivo after temperature treatment}

To characterize the induction of Bitxl gene in response to external temperature stress, B. ignitus workers were exposed to $4^{\circ} \mathrm{C}$ or $37^{\circ} \mathrm{C}$ for $6 \mathrm{~h}$, respectively, while controls were maintained at $27^{\circ} \mathrm{C}$. The induction of Bitxl in the fat body was analyzed by Northern blot. As shown in Fig. 5, the level of Bitxl in fat body significantly increased after exposure to low or high temperature

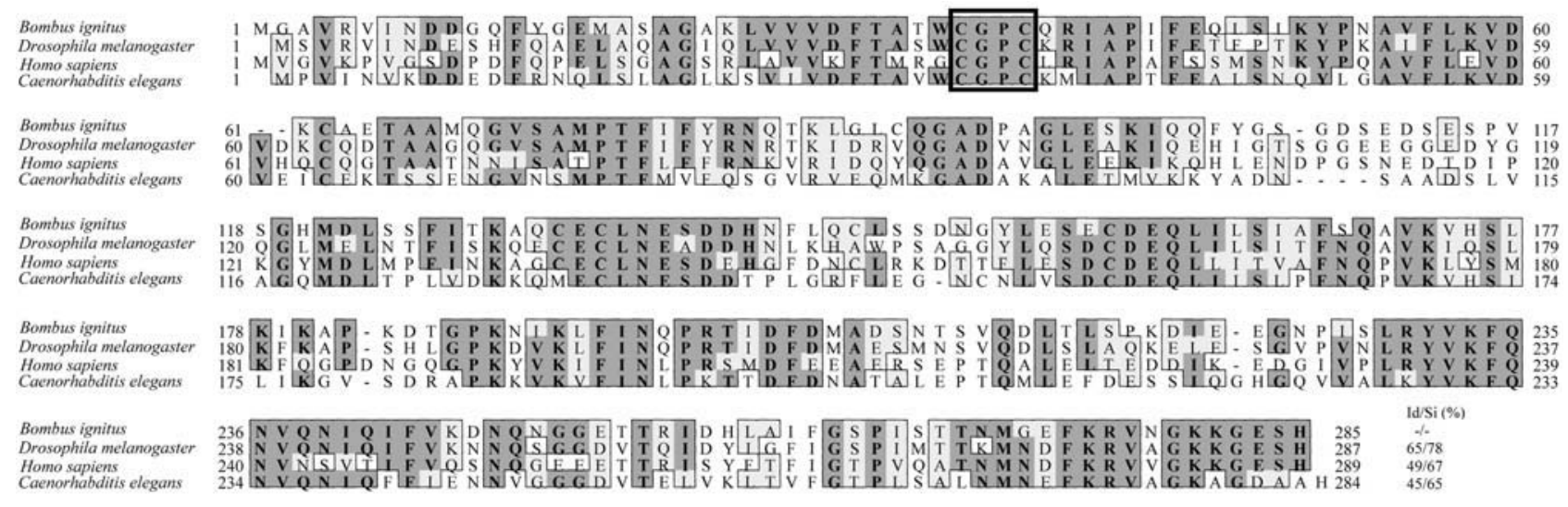

Fig. 2. Comparison of the deduced amino acid sequence of Bitxl with that of other txls. Invariant residues are shaded black. The txl active site is boxed. GenBank accession numbers are: D. melanogaster (AF143404), C. elegans (AF143405) and human (NM004786). Bitxl sequence was used as a reference for the identity / similarity ( $\mathrm{Id} / \mathrm{Si}$ ) values. 


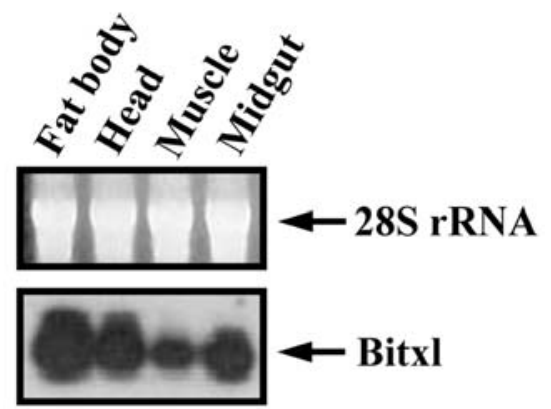

Fig. 3. Bitxl mRNA expression in B. ignitus tissues. Total RNA was isolated from the fat body, head, muscle and midgut, respectively (upper panel). The RNA was separated by $1.0 \%$ formaldehyde agarose gel electrophoresis, transferred on to a nylon membrane and hybridized with radiolabelled 855 bp Bitxl cDNA (lower panel). Transcripts are indicated on the right side of the panel by an arrow.

stress, compared with the control. After each temperature treatment, the level of Bitxl was significantly increased after $1 \mathrm{~h}$, remained at a high level from $2-4 \mathrm{~h}$ and then declined. The result indicates that Bitxl is up-regulated by low and high temperature shocks.

\section{$T x l$ expression in vivo after lipopolysaccharide (LPS) injection}

To assess the induction of the Bitxl gene after LPS injection, $B$. ignitus workers were injected with LPS.

(A)

$\mathrm{H}_{2} \mathrm{O}_{2}$

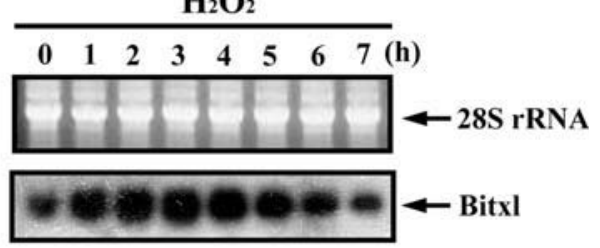

(B)

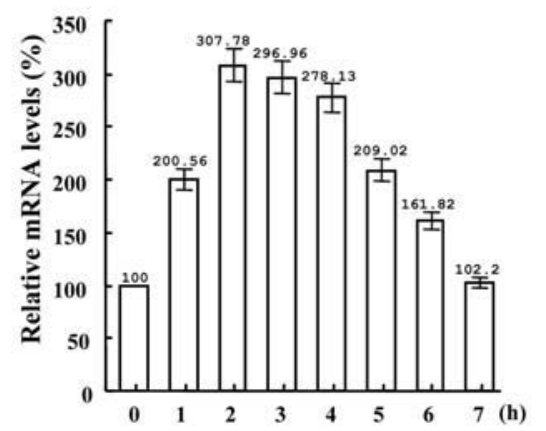

Fig. 4. Induction of Bitxl by in vivo injection of $\mathrm{H}_{2} \mathrm{O}_{2}$. (A) Northern blot analysis of the Bitxl gene induced by $\mathrm{H}_{2} \mathrm{O}_{2}$ injection. The B. ignitus workers were injected with $10 \mathrm{mM} \mathrm{H}_{2} \mathrm{O}_{2}$. Total RNA was isolated from the fat body of $B$. ignitus workers at $1 \mathrm{~h}$ intervals. The RNA was separated by $1.0 \%$ formaldehyde agarose gel electrophoresis (upper panel), transferred on to a nylon membrane and hybridized with radiolabelled 855 bp Bitxl cDNA (lower panel). Transcripts are indicated on the right side of the panel by an arrow. (B) Relative mRNA levels of Bitxl induced by $\mathrm{H}_{2} \mathrm{O}_{2}$ injection. Relative mRNA levels of Bitxl are means of three assays, which are calculated relative to that of the expression recorded for the control (shown as 100\%). Bars represent the means plus/minus SE.
(A)

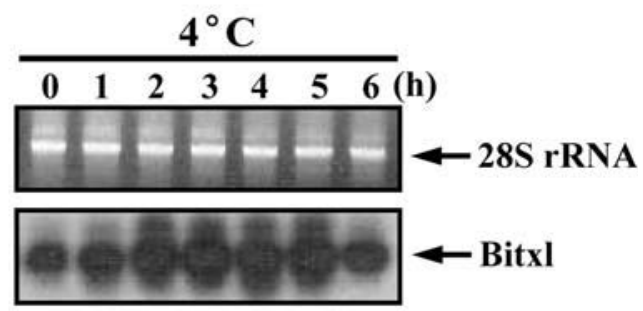

(B)

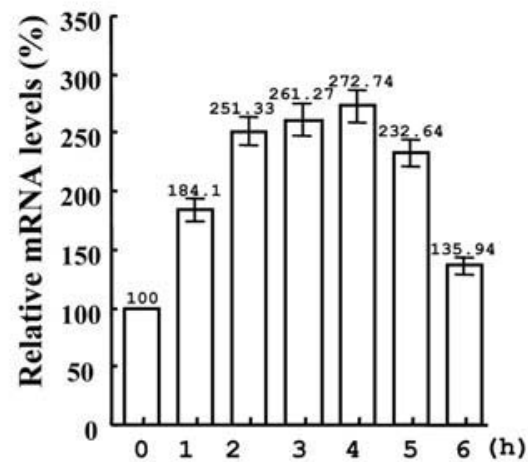

(C) $37^{\circ} \mathrm{C}$

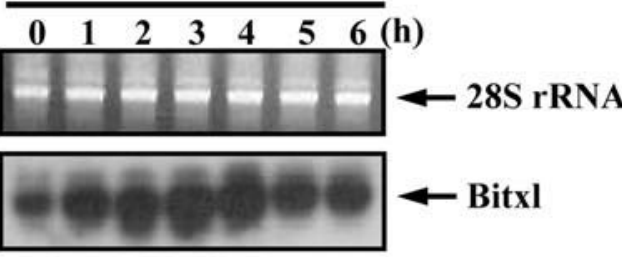

(D)

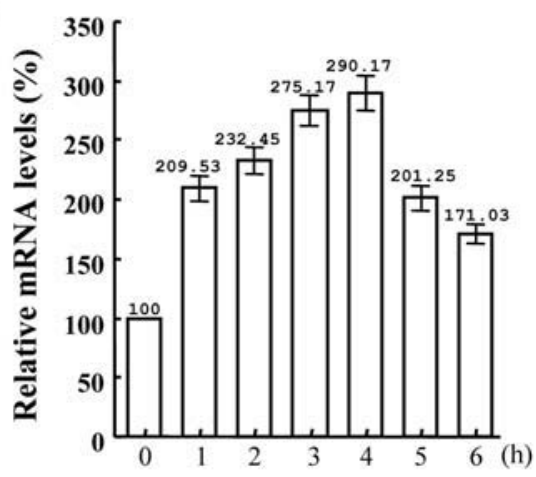

Fig. 5. Induction of Bitxl by external temperature stress. The B. ignitus workers were incubated at $4^{\circ} \mathrm{C}(\mathrm{A}$ and $\mathrm{B})$ or $37^{\circ} \mathrm{C}(\mathrm{C}$ and D) for $6 \mathrm{~h}$, respectively. Controls were kept indoors at $27^{\circ} \mathrm{C}$ (lane 0 of each panel). Total RNA was isolated from the fat body of $B$. ignitus workers kept at each temperature. The RNA was separated by $1.0 \%$ formaldehyde agarose gel electrophoresis (upper panel of $\mathrm{A}$ and $\mathrm{C}$ ), transferred on to a nylon membrane and hybridized with radiolabelled 855 bp Bitxl cDNA (lower panel of A and C). Transcripts are indicated on the right side of the panel by arrows. Relative mRNA levels of Bitxl induced by the $4^{\circ} \mathrm{C}$ (B) and $37^{\circ} \mathrm{C}$ (D) treatments were measured. Relative mRNA levels of Bitxl are means of three assays, which are calculated relative to this expression at $27^{\circ} \mathrm{C}$ (shown as $100 \%$ ). Bars represent the means plus/minus SE. 
(A)

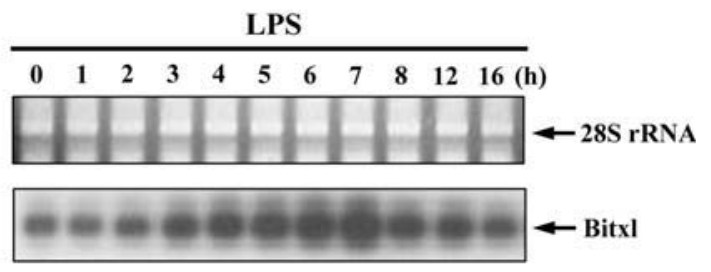

(B)

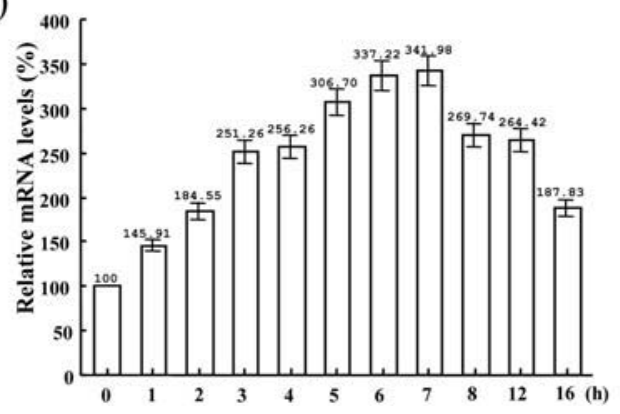

Fig. 6. Induction of Bitxl by in vivo injection of LPS. (A) Northern blot analysis of the Bitxl gene induced by LPS injection. The $B$. ignitus workers were injected with $10 \mathrm{mM}$ LPS Total RNA was isolated from the fat body of B. ignitus at intervals of 1 to $16 \mathrm{~h}$. The RNA was separated by $1.0 \%$ formaldehyde agarose gel electrophoresis (upper panel), transferred on to a nylon membrane and hybridized with radiolabelled $855 \mathrm{bp}$ Bitxl cDNA (lower panel). Transcripts are indicated on the right side of the panel by arrows. (B) Relative mRNA levels of Bitxl induced by LPS injection. Relative mRNA levels of Bitxl are means of three assays, which are calculated relative to the level in the control (shown as $100 \%$ ). Bars represent the means plus/minus SE.

After injection, total RNA was isolated from the fat body of the B. ignitus workers. The Bitxl in the fat body induced by LPS injection was analyzed by Northern blot. The level of Bitxl mRNA in fat body significantly increased in treated workers, compared with the control (Fig. 6). The Bitxl mRNA expression peaked $7 \mathrm{~h}$ after LPS stimulation, indicating that Bitxl is up-regulated by LPS injection. Furthermore, Bitxl expression level induced by LPS stimulation $5-7 \mathrm{~h}$ after injection was more than 3 times more in treated than in the control workers.

\section{DISCUSSION}

In this study, the B. ignitus txl gene was cloned and characterized to elucidate the physiological role of txl in insects. The Bitxl gene consisted of three introns and four exons coding for 285 amino acid residues. The conserved active site residues in Bitxl are present at the same positions as those in txls from other species, in which these residues are involved in thioredoxin activity (MirandaVizuete et al., 1998; Miranda-Vizuete \& Spyrou, 2000). Bitxl was closest in structure to D. melanogaster txl $(65 \%$ protein sequence identity). Critical conserved residues for optimal thioredoxin activity are present in both D. melanogaster and C. elegans txls but absent in the human txl
(Miranda-Vizuete \& Spyrou, 2000). The critical conserved residues are present in the Bitxl protein, except for Lys36. The residue flanking the active site, Lys36, which stabilizes the thiolate in the active site (Eklund et al., 1991), is a Gln residue. On the basis of these characteristics, it is proposed that Bitxl is one of the expanding family of thioredoxins.

Txl is a cytosolic ubiquitously expressed protein and it has been copurified with a kinase of the STE20 family, which is proteolytically activated by caspases in apoptosis (Lee et al., 1998; Miranda-Vizuete et al., 1998; MirandaVizuete \& Spyrou, 2000). However, no cellular function has yet been assigned to this protein. The expression of Bitxl at the transcriptional level revealed that Bitxl transcripts were present in all tissues examined, indicating that Bitxl is ubiquitously expressed. Furthermore, the fat body of $B$. ignitus workers injected with $\mathrm{H}_{2} \mathrm{O}_{2}$ showed an increase in the transcript level of Bitxl. This result suggests that Bitxl plays an important role in protecting tissues against oxidative damage caused by intracellularly generated ROS during metabolism.

Most organisms are sensitive to sudden temperature stress. Extreme temperatures are a major stress faced by all organisms. It is reported that temperature stress is a key mediator of the formation of ROS (Hariari et al., 1989; Rauen et al., 1999). In previous studies, insect enzymes, Gryllotalpa orientalis SOD1 (Kim et al., 2005b), Bombyx mori TPx (Lee et al., 2005) and G. orientalis Prx (Kim et al., 2005a), were up-regulated by bolt cold and heat stress. In this study, Bitxl induction by temperature stress, a mediator of ROS, suggests that Bitxl may play an important role as an antioxidant protein, by reducing the high level of intracellular hydrogen peroxide induced by extracellular stimuli such as low or high temperatures.

It is well known that LPS mediates many pathophysiological events in insects by stimulating the release of host-derived antibacterial proteins (Hartmann \& Krieg, 1999; Lindmark et al., 2001; Korner \& Schmid-Hempel, 2004). In this study, the induction of Bitxl in B. ignitus workers after LPS injection was demonstrated. It is likely that the up-regulation of Bitxl is related to its role in protecting against oxidative damage caused by LPS stimulation. In another insect, the antioxidant protein, B. mori TPx, was significantly induced during viral infection (Lee et al., 2005). Furthermore, B. ignitus SOD1 was upregulated by LPS stimulation (Choi et al., in prep.). In light of this, our present results suggest that the upregulation of Bitxl by LPS stimulation may indicate its involvement in the protection against bacterial infection.

In conclusion, this study was undertaken to search for a homologue of txl in B. ignitus and to elucidate its functional role in antioxidant defense. The data presented suggest that Bitxl is an insect antioxidant protein, which is ubiquitously expressed and functions in antioxidant defense. The fact that the Bitxl in the fat body of $B$. ignitus was up-regulated by $\mathrm{H}_{2} \mathrm{O}_{2}$, temperature stress or LPS stimulation suggests that it has an important role in 
the protection against oxidative damage caused by temperature stress or bacterial infection.

ACKNOWLEDGEMENTS. This work was funded by the Dong-A University Research Fund.

\section{REFERENCES}

Altschul S.F., Madden T.L., Schaffer A.A., Zhang J., Zhang Z., Miller W. \& Lipman DJ. 1997: Gapped BLAST and PSIBLAST: a new generation of protein database search programs. Nucl. Acids Res. 25: 3389-3402.

Araki M., Nanri H., Ejima K., Murasato Y., Fujiwara T., NaKashima Y. \& IKeda M. 1999: Antioxidant function of the mitochondrial protein SP-22 in the cardiovascular system. $J$. Biol. Chem. 274: 2271-2278.

Arner E.S. \& Holmgren A. 2000: Physiological functions of thioredoxin and thioredoxin reductase. Eur. J. Biochem. 267: 6102-6109.

Bryk R., Lima C.D., Erdjument-Bromage H., Tempst P. \& NAtHAn C. 2002: Metabolic enzymes of mycobacteria linked to antioxidant defense by a thioredoxin-like protein. Science 295: $1073-1077$.

Choi Y.S., Lee K.S., Yoon H.J., Kim I., Sohn H.D. \& Jin B.R.: A bumblebee $\mathrm{Cu}, \mathrm{Zn}$ superoxide dismutase (SOD1) that is upregulated on temperature stimulus and lipopolysaccharide injection. Comp. Biochem. Physiol. (B) (submitted).

Eklund H., Gleason F.K. \& Holmgren A. 1991: Structural and functional relations among thioredoxins of different species. Proteins 11: 13-28.

Hariari P.M., Fuller D.J. \& Gerner E.W. 1989: Heat shock stimulates polyamine oxidation by two distinct mechanisms in mammalian cell cultures. Int. J. Radiat. Oncol. Biol. Phys. 16: $451-457$.

Hartmann G. \& Krieg A.M. 1999: CpG DNA and LPS induce distinct patterns of activation in human monocytes. Gene Ther. 6: 893-903.

Holmgren A. 1985: Thiredoxin. Annu. Rev. Biochem. 54: 237-271.

Holmgren A. 1989: Thioredoxin and glutaredoxin systems. $J$. Biol. Chem. 264: 13963-13966.

Kim I., Lee K.S., Hwang J.S., Ahn M.Y., Li J., Sohn H.D. \& JiN B.R. 2005a: Molecular cloning and characterization of a peroxiredoxin gene from the mole cricket, Gryllotalpa orientalis. Comp. Biochem. Physiol. (B) 140: 579-587.

Kim I., Lee K.S., Hwang J.S., Sohn H.D. \& Jin B.R. 2005b: Molecular cloning and characterization of the $\mathrm{Cu}, \mathrm{Zn}$ superoxide dismutase (SOD1) gene from the mole cricket, Gryllotalpa orientalis. Biotechnol. Lett. 27: 589-595.

Ko F.C.F. \& Chow K.L. 2002: A novel thioredoxin-like protein encoded by the C. elegans dpy-11 gene is required for body and sensory organ morphogenesis. Development 129: $1185-1194$.

Koner P. \& Schmid-Hempel P. 2004: In vivo dynamics of an immune response in the bumble bee Bombus terrestris. $J$. Invertebr. Pathol. 87: 59-66.
Kurooka H., Kato K., Minoguchi S., Takahashi Y., Ikeda J.E., Habu S., Osawa N., Buchberg A.M., Moriwaki K., Shisa H. \& HonJo T. 1997: Cloning and characterization of the nucleoredoxin gene that encodes a novel nuclear protein related to thioredoxin. Genomics 39: 331-339.

Laurent T.C., Moore E.C. \& Reichard P. 1964: Enzymatic synthesis of deoxyribonucleotides. IV. Isolation and characterization of thioredoxin, the hydrogen donor from Escherichia coli B. J. Biol. Chem. 239: 3436-3444.

Lee K.K., Murakawa M., Takahashi S., Tsubuki S., Kawashima S., Sakamaki K. \& Yonehara S. 1998: Purification, molecular cloning, and characterization of TRP32, a novel thioredoxinrelated mammalian protein of $32 \mathrm{kDa}$. J. Biol. Chem. 273: 19160-19166.

Lee K.S., Kin S.R., Park N.S., Kim I., Kang P.D., Sohn B.H., Chor K.H., Kang S.W., Je Y.H., Lee S.M., Sohn H.D. \& JIN B.R. 2005: Characterization of a silkworm thioredoxin peroxidase that is induced by external temperature stimulus and viral infection. Insect Biochem. Mol. Biol. 35: 73-84.

Lindmark H., Johansson K.C., Stoven S., Hultmark D., EnGStrom Y. \& Soderhall K. 2001: Enteric bacteria counteract lipopolysaccharide induction of antimicrobial peptide genes. J. Immunol. 167: 6920-6923.

Matsuo Y., Akiyama N., Nakamura H., Yodoi J., Noda M., KIZAKA-KoNDOH S. 2001: Identification of a novel thioredoxin-related transmembrane protein. J. Biol. Chem. 276: $10032-10038$.

McMaster G.K. \& CARmichael G.G. 1977: Analysis of singleand double-stranded nucleic acids on polyacrylamide and agarose gels by using glyoxal and acridine orange. Proc. Natl. Acad. Sci. USA 74: 4835-4838.

Miranda-Vizuete A. \& Spyrou G. 2000: Genomic structure and chromosomal localization of human thioredoxin-like protein gene (txl). DNA Seq. 10: 419-424.

Miranda-Vizuete A., Gustafsson J.A. \& Spyrou G. 1998: Molecular cloning and expression of a cDNA encoding a human thioredoxin-like protein. Biochem. Biophys. Res. Commun. 243: 284-288.

NaKamura H. 2005: Thioredoxin and its related molecules. Antioxid. Redox Signal. 7: 823-828.

Rauen U., Polzar B., Stephan H., Mannherz H.G. \& de Groot H. 1999: Cold-induced apoptosis in cultured hepatocytes and liver endothelial cells: mediation by reactive oxygen species. FASEB J. 13: 155-168.

Spyrou G., Enmark E., Miranda-Vizuete A. \& Gustafsson J.A. 1997: Cloning and expression of a novel mammalian thioredoxin. J. Biol. Chem. 272: 2936-2941.

Yoon H.J., KIM S.E. \& KIM Y.S. 2002: Temperature and humidity favorable for colony development of the indoorreared bumblebee, Bombus ignitus. Appl. Entomol. Zool. 37: 419-423.

Yoon H.J., Kim S.E. \& Kim Y.S. \& LeE S.B. 2004: Colony developmental characteristics of the bumblebee queen, Bombus ignitus by the first oviposition day. Int. J. Indust. Entomol. 8: 139-143.

Received August 10, 2005 revised and accepted September 12, 2005 